\title{
Thermodynamics and Irreversibility: From Some Paradoxes to the Efficiency of Effective Engines
}

\author{
Olivier Serret \\ ESIM Engineer-60 rue de la Marne, Cugnaux, France \\ Email: o.serret@free.fr
}

Received 5 August 2014; revised 2 September 2014; accepted 27 September 2014

Copyright (C) 2014 by author and Scientific Research Publishing Inc.

This work is licensed under the Creative Commons Attribution International License (CC BY).

http://creativecommons.org/licenses/by/4.0/

(c) (i)

\begin{abstract}
The traditional thermodynamic theory explains the reversible phenomena quite well, except that reversible phenomena are rare or even impossible in practice. Here the purpose is to propose an explanation valid for reversible and also irreversible phenomena, irreversibility being common or realistic. It previously exposed points tricky to grasp, as the sign of the work exchange, the adiabatic expansion in vacuum (free expansion) or the transfer of heat between two bodies at the same temperature (isothermal transfer). After having slightly modified the concepts of heat transfer (each body produces heat according to its own temperature) and work (distinguishing external pressure from internal pressure), the previous points are more easily explained. At last, an engine efficiency in case of irreversible transfer is proposed. This paper is focused on the form of thermodynamics, on "explanations"; it does not question on "results" (except the irreversible free expansion of 1845 ...) which remain unchanged.
\end{abstract}

\section{Keywords}

Heat Transfer, External and Internal Pressure Work, Internal Energy, Reversible and Irreversible, Joule's Law and Joule's Experiments, Adiabatic and Free Expansion, Clapeyron Diagram, Carnot Cycle, Engine Efficiency

\section{Introduction}

Thermodynamics traditionally [1] [2] deals of relations between the thermal and the mechanical phenomena. It is a science that was born in the nineteenth century and was codified by scholars as Sadi CARNOT or James Prescott JOULE. Since then, little major changes have been made, on form and content. It is on this first point that presentation takes place in this essay. Indeed, some statements remain difficult to understand, even if current 
"explanations" do not taint the veracity of the "results".

In the first part, we will check some statements tricky to understand: a mathematical equality considered as a physical principle, an expansion without any work, a negative work represented by a positive area, a transfer of heat between two bodies at the same temperature, and a cycle where heat seems to go from cold to hot! In the second part, we will change the forms of the heat transfer and of the work and consequently of the internal energy. Then in the third part, we will apply these new forms to the first statements which were tricky to understand. Explanations look then more understandable, especially for irreversible phenomena. At last, an efficiency ratio based on differences of temperatures is proposed for irreversible cycles.

\section{Some Statements Tricky to Understand}

\subsection{The "Principle Zero" of Thermodynamics}

Its name or more precisely its numbering- "zero"-is unusual. The reason is historical. The first principles having already been laid, it has nevertheless been considered necessary to add another one prior to the demonstrations. But more than its numbering, it is its content which is surprising as a "principle". Recall that the principle zero states that if $A$ is in (thermal) equilibrium with $B$ and $B$ with $C$, then $A$ is with $C$. This is surprising because that looks like more to a mathematical property (a transitive law, an equivalence relation or an axiom as "two quantities equal to a third are equal") than to a physical principle. In other branches of physics, it is not stated as a "principle" that if $A$ has the same mass as $B$ and $B$ as $C$, then $A$ has the same mass as $C$. This zero principle asks a question: does not it reflect a certain difficulty in characterizing thermal equilibrium?

\subsection{Free Expansion and Joule Experiment}

\subsubsection{Historical}

In 1806, Louis GAY-LUSSAC was the first to experiment expansion in vacuum. Measuring the temperature directly on the air with an alcohol thermometer, he found no variation of temperature [3].

In 1845, James JOULE did the same experiment, except that it measured the temperature of the surrounding water; he found no variation of temperature either [4].

In 1865, Gustave-Adolphe HIRN did the same experiment, measuring the temperature through a variation of pressure. He found a light variation of temperature: $-0.2^{\circ} \mathrm{C}$ [5]. See Figure 1.

Since there, the experiment has been done again with carbon dioxide $\mathrm{CO}_{2}$ (which is more sensitive than air): it has been noted a decrease of temperature of $-0.3^{\circ} \mathrm{C}[6]$ [7].

And today, it is mainly the usual industrial way to cool gases [8] [9].

And yet History has retained the result of the Joule's experiment with no variation of temperature!

\subsubsection{Explanation}

Let us remind that an ideal gas is a gas that obeys the ideal gas law:

$$
P \cdot V=n \cdot R \cdot T
$$

with $P$ : internal pressure of the gas; $V$ : volume of gas; $n$ : amount of substance of the gaz (in moles); $R$ : gaz constant $\left(8.314 \mathrm{~J} \cdot \mathrm{K}^{-1} \cdot \mathrm{mol}^{-1}\right)$; $T$ : absolute temperature (in Kelvin).

This is a good approximation for gases in usual conditions (when the pressure is relatively low, less than 10 atm.) like the atmospheric pressure. It is often used to describe cycles, compressions and expansions.

There are two laws of Joule: the first one called Joule-Gay Lussac law and the second one called JouleThomson law. The first law states that for ideal gas the internal energy depends only on the temperature, the

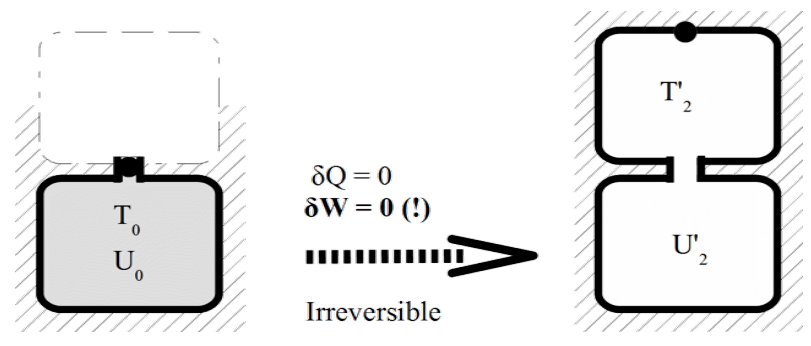

Figure 1. The so-called Joule’s experiment. 
second one that the enthalpy depends only on its temperature. To check it, these scholars set up an experiment showing that "the gas temperature would remain constant" (which is quite different to the Joule's law: $U$ depends only on its temperature) with the expansion, due to the formula

$$
\mathrm{d} U=\delta Q+\delta W
$$

The reasoning is as follow: because there is no heat transfer, and because there is no work ( $P_{\text {vacuum }}=0$ so without external pressure, there would not be pressure work), there is no change of internal energy $U$ and so we should not have change of temperature.

This is why it is still traditionally explained that if the gas cools in experiments, it is that in sudden expansion the gas is far to be ideal [the gas would not obey to Equation (1)]: this is an indirect recognition that traditional theory is not in agreement with experimental results...

\subsubsection{A Surprising Result}

What a surprising result: a thermodynamic system which keeps constant its internal energy $U$ and its temperature $T$, and which does not receive any heat or work but submits an irreversible change! It is the result found by Pr. Joule in 1845.

Why is it qualified here of surprising result? Because it is unusual to get:

- no work when the volume changes; it would be surprising that the border line, which can be the black top of Figure 1, moves without any work! It would be at variance with the first principle of thermodynamics where perpetual motion machines of the first kind are impossible...

- a constant temperature

$$
T_{2}^{\prime}=T_{0}
$$

when in the reversible and in the other irreversible cases, the temperature decreases [10] with expansion (without heat transfer); please check on Figure 2.

\subsection{Work Representation by Its Area}

In mathematics, the area is calculated by integrating a function. If the function $f_{\text {math }}(x)$ is positive so its integral, represented by an area, is positive: $\left[F_{\text {math }}\right]>0$ [11]. See Figure 3 where $f_{\text {math }}(x)$ is $P_{\text {ext }}(V)$ and $\left[F_{\text {math }}\right]$ is $\left[W_{\text {math }}\right]$.

In thermodynamics, work is conventionally counted positively when received and negatively when provided by the system. The infinitesimal work exchanged by the system is denoted $\delta \mathrm{W}$.

$$
\delta W=-P_{\text {ext }} \mathrm{d} V
$$

The pressure $P_{\text {ext }}$ being always positive, when $V_{2}$ is higher than $V_{1}$ (that is, when $x_{2}$ higher than $x_{1}$ ), the thermodynamics sum $W$ is then negative: $W<0$. How explain that in the [P-V] Clapeyron diagram particularly suitable for representation of work, the negative work $W$ is paradoxically represented by a positive area $W_{\text {math }}$ ?

\subsection{Carnot Cycle}

The theorizing of thermodynamics by physicists did not precede but followed or accompanied the development by engineers of thermal engines as the steam engine. They had already realized that the efficiency $\eta$ (work

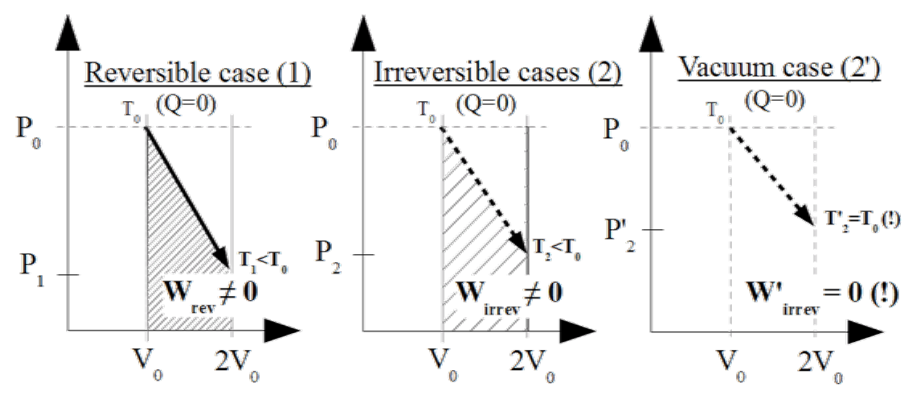

Figure 2. Work and temperature evolution with expansion. 


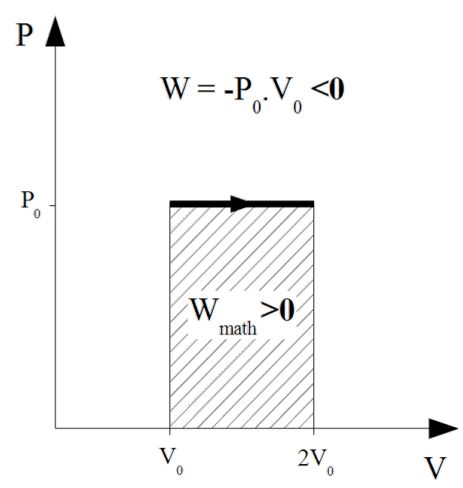

Figure 3. Representation of positive mathematical area.

done/energy transmitted by the hot source) seemed limited by an unsurpassable value related to the temperatures of the sources.

$$
\eta \leq 1-\frac{T_{\text {Cold }}}{T_{\mathrm{Hot}}}
$$

Was it an impression or a physical principle? Sadi Carnot, who thought the heat was a fluid called caloric, described a clever cycle (not representative of an existing machine) which reaches the performance limit. Let us consider a transformation of this cycle, the isothermal transformation. See Figure 4.

An isothermal transformation is a transformation that takes place at constant temperature, in contact with a thermostat. Take for example a piston air-filled at $20^{\circ}$ Celsius, in an external medium like water also at $20^{\circ}$ Celcius. The air volume is adapted to the pressure according to the ideal gas law. This system is in stable equilibrium; it does not change and will not change spontaneously. And there is no reason that for two bodies at the same temperature, heat flows in one direction rather than another. In the case of perfect equality between fluid temperature and thermostat temperature: there is no transfer of heat.

$$
Q_{\text {Hot }}=Q_{\text {Cold }}=0
$$

And more generally for isothermal transformation

$$
T_{\text {fluid }}=T_{\text {thermostat }} \rightarrow Q=0
$$

This cycle cannot work as a thermal engine.

The efficiency $\eta$ of the Carnot cycle is defined as the ratio of work supplied of the heat input, (i.e. by energy conservation), dividing heat balance by heat input:

$$
\eta=\frac{Q_{\mathrm{Hot}}-Q_{\mathrm{Cold}}}{Q_{\mathrm{Hot}}}
$$

Hence [cf Equation (6)]

$$
\eta=\frac{0}{0}
$$

$\eta$ is not calculable. Rigorously, the Carnot efficiency (based on isothermal conditions) is not defined!

\subsection{Carnot Engine}

The thermal cycle of a motor is traditionally represented as follows in Figure 5.

In motor mode, the heat from the hot source is partly converted in form of work, the balance going to the cold source.

And the thermal cycle of a refrigerating unit is traditionally represented as follows in Figure 6.

In refrigeration mode, the heat from the cold source goes to the hot source and the work too. How is it possible that the heat goes from cold to hot? 


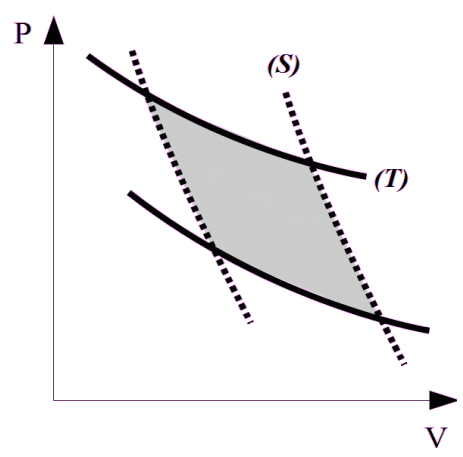

Figure 4. Carnot cycle.
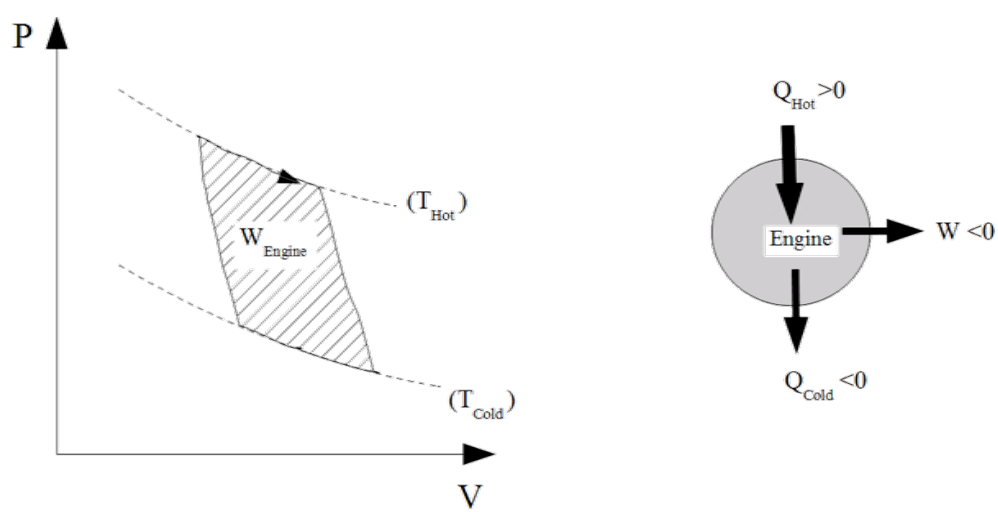

Figure 5. Traditional representations of motor cycle.
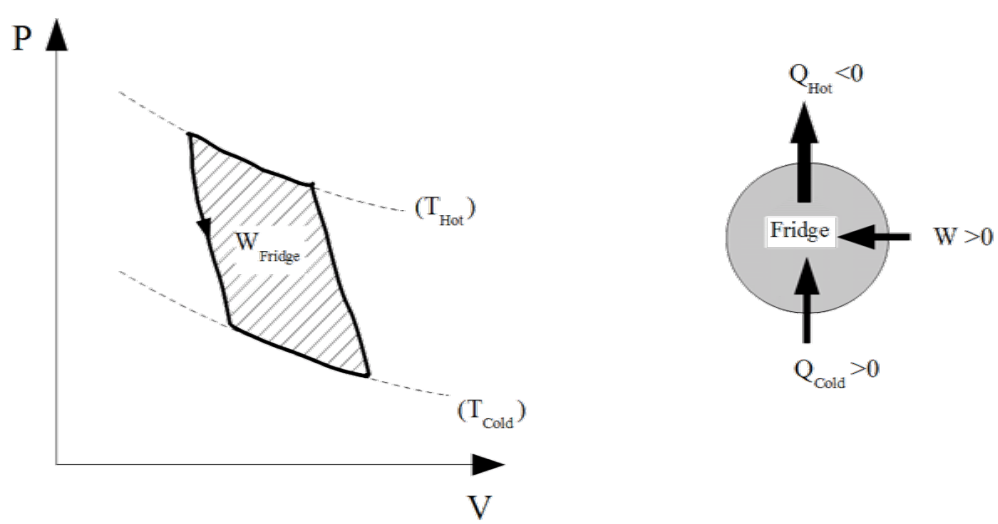

Figure 6. Traditional representations of cooling cycle.

\section{Statement of Assumptions}

The above points will be discussed again after the following assumptions:

\subsection{Internal Energy $U$}

Beforehand, what is the internal energy $U$ ? “The internal energy of given state cannot be directly measured. ( $\cdots)$ Though it is a macroscopic quantity, internal energy can be explained in microscopic terms by two theoretical virtual components. One is the microscopic kinetic energy $(\cdots)$. The other is the potential energy $(\cdots)$. There is no simple universal relation between these quantities of microscopic energy and the quantities of energy gained or lost by the system in work, heat, or matter transfer.” [12]. So the link between macroscopic and virtual microscopic components is: 


$$
U=\sum\left(E_{K}\right)+\sum\left(E_{P}\right)
$$

with $E_{K}$ for kinetic energy of particles and $E_{P}$ for potential energy

And the main property of $U$ is: "The internal energy is a state function of the system, because its value depends only of the current state of the system and not on the path taken or processes undergone to prepare it." [8]. This property does not change here.

\subsection{Emitted Heat Proportional to Temperature}

The first new assumption is that all bodies emit heat, and this heat $\delta Q$ is proportional to the temperature $T$ of the body over a period $\mathrm{d} t$ :

$$
\delta Q_{\text {emitted }}=s \cdot A \cdot T \cdot \mathrm{d} t
$$

with:

- $s$ coefficient, unit in $\left[\mathrm{W} /\left(\mathrm{K} \cdot \mathrm{m}^{2}\right)\right]$

- A area of the body, in $\left[\mathrm{m}^{2}\right]$

- $T$ temperature of the body surface, in [K]

- $\mathrm{d} t$ time length of the thermal exchange, in [s]

Remark: if entropy $S$ in $[\mathrm{J} / \mathrm{K}]$ has the properties of a status function, $s$ is only a coefficient in $\left[\mathrm{W} /\left(\mathrm{K} \cdot \mathrm{m}^{2}\right)\right]$. And like $C_{p}, s$ is empirical and should be dependent for example on the temperature.

\subsection{Work of Internal Pressure}

According to the fundamental principle of dynamics, acceleration undergone by a body is proportional to the net strength it received. The piston is moved because at a time that internal strength was different from the external strength, i.e. the internal pressure was different from the external pressure. See Figure 7.

It is considered here (please read arguments in Appendix) the internal strength or internal pressure, and therefore their work according to the formula:

$$
\delta W_{\text {int }}=F_{\text {int }} \cdot \mathrm{d} l
$$

or

$$
\delta W_{\text {int }}=P_{\text {int }} \cdot \mathrm{d} V
$$

Remark: The fundamental principle of dynamics indicates that if internal pressure equals constantly and exactly to external pressure, then there is no movement (for a body initially at rest): the reversible movement where constantly and exactly $P_{\text {int }}=P_{\text {ext }}$ is impossible. The reversible movement is a limit but an unrealistic case.

\subsection{Variation of Internal Energy Function of Heat Transfer and Internal Pressure Work}

The property to calculate the "variation" of $U$ is traditionally given by $\mathrm{d} U=\delta Q+\delta W \quad$ [cf Equation (2)] with $\delta W=P_{\text {ext }} \cdot\left(-\mathrm{d} V_{\text {int }}\right)$ [cf Equation (4)] which gives.

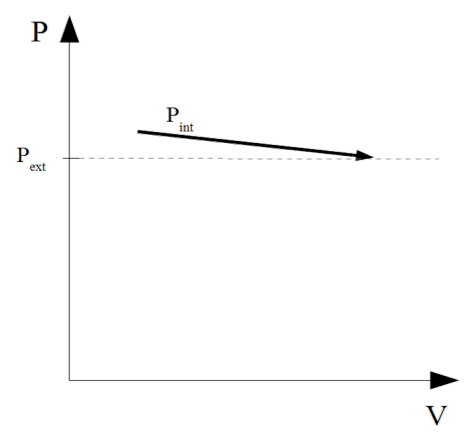

Figure 7. $P_{\text {int }}$ was different from $P_{\text {ext }}$. 


$$
\mathrm{d} U=\delta Q-P_{\text {ext }} \cdot \mathrm{d} V
$$

Note than the variation of the "internal" energy would depend from the "external" pressure.

In this essay let us rather have the internal energy to depend from the internal pressure:

$$
\mathrm{d} U=\delta Q-\delta W_{\text {int }}
$$

or

$$
\mathrm{d} U+\delta W_{\text {int }}=\delta Q
$$

with $\delta W_{\text {int }}=P_{\text {int }} \cdot \mathrm{d} V_{\text {int }} \quad[$ cf Equation (13)] which means

$$
\mathrm{d} U=\delta Q-P_{\text {int }} \cdot \mathrm{d} V
$$

\subsection{Comments}

- $P_{\text {ext }}$ has only been changed into $P_{\text {int }}$, there is no major change in the expression of $|\delta W|$ and dU; no major change means that $P_{\text {int }}$ has in practice the same magnitude than $P_{\text {ext }}$ (except of course in the particular case of expansion in vacuum).

- When $P_{\text {int }}=P_{\text {ext }}$, then $\mathrm{d} U$ according to Equation (14) is equal to $\mathrm{d} U$ according to Equation (16):

$$
\delta Q+P_{\text {ext }} \cdot(-\mathrm{d} V)=\delta Q-P_{\text {int }} \cdot \mathrm{d} V
$$

- For reversible paths, $P_{\text {int }}=P_{\text {ext }}$, so there is no difference in the calculation of $\mathrm{d} U$.

- For irreversible paths, we will get a difference in the calculation of $|\delta W|$. This difference of work calculation will change the "predicted" reached point in the Clapeyron diagram; for example, shall we reach the temperature $T_{1}$ or $T_{2}$ ? See Figure 8.

- Then, to calculate the difference of internal energy from the initial point $\left(T_{0}\right)$, we will choose a "reversible" path, and we have demonstrated herebefore there was no difference in the calculation of $\mathrm{d} U_{\text {rev }}$, and so in the calculation of the $U$ status [we will find either $U\left(T_{1}\right)$ or $U\left(T_{2}\right)$ ].

- It confirms the $U$ value of such defined point depends only of the current state of the system and not on the path taken or processes undergone to prepare it.

- For ideal gas, the property that the macroscopic internal energy $U$ depending only on the temperature (the Joule's law) remains unchanged.

- Due to Equation (10) and Equation (15bis), the link between the macroscopic value $U$ and the virtual microscopic terms could be changed.

To sum up into a chart (Chart 1).

\section{Consequences}

In function of these hypotheses, points of Paragraph 2 are now discussed again.

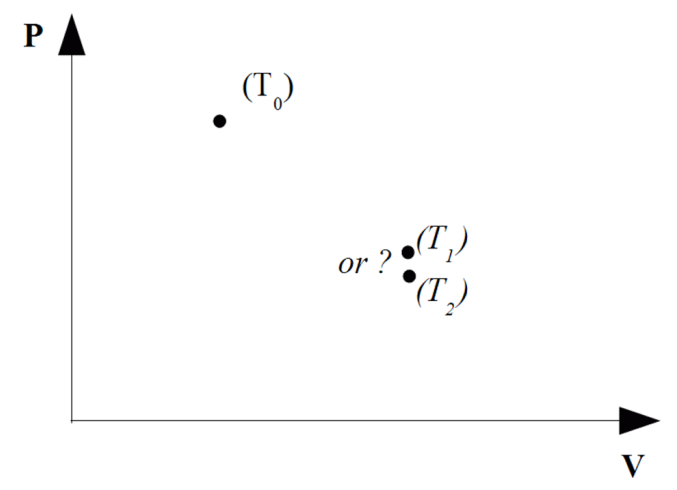

Figure 8. Change of the predicted reached point. 


\subsection{Thermal Balance and Heat Transfer}

\subsubsection{Concept}

Suppose two identical bodies, except for temperature, in a fully insulated enclosure: all the heat emitted from one body $A$ is received by the other body $B$ (and vice versa). According to the first hypothesis of Equation (11) where $\delta Q_{\text {emitted }}$ is proportional to temperature, at every moment (see Figure 9):

- the body $A$ at the temperature $T_{A}$ emits the heat $\delta Q_{\text {emitted/A }}$ and receives from $B$ the heat $\delta Q_{\text {emitted/B }}$

- the body $B$ at the temperature $T_{B}$ emits the heat $\delta Q_{\text {emitted } / B}$ and receives from $A$ the heat $\delta Q_{\text {emitted/A }}$

- For $A$, the balance between the emitted heat and the received heat is the heat $\delta Q_{\text {balance/A }}$ :

$$
\delta Q_{\text {balance/A }}=\delta Q_{\text {emitted } / B}-\delta Q_{\text {emitted } / A}
$$

- For $B$, the balance between the emitted heat and the received heat is the heat $\delta Q_{\text {balance/B }}$ :

$$
\delta Q_{\text {balance } / B}=\delta Q_{\text {emitted/A }}-\delta Q_{\text {emitted/B }}
$$

This heat $\delta Q_{\text {balance }}$ is nothing but the exchanged heat $\delta Q$ in the traditional theory.

\subsubsection{Generalization}

- We can note in the case of this single two bodies that

$$
\delta Q_{\text {balance } / B}=-\delta Q_{\text {balance } / \mathrm{A}}
$$

This is in agreement with energy conservation, what is lost from one side is gained from the other side.

- If for example $T_{A}>T_{B}$, then according to Equation (11) on emitted heat

$$
\delta Q_{\text {emitted } / A}>\delta Q_{\text {emitted/B }}
$$

and so

$$
\delta Q_{\text {balance/A }}<0
$$

\begin{tabular}{|c|c|c|c|}
\hline & Theory & Traditional & Proposed \\
\hline \multirow{3}{*}{$U$} & Determination & Cannot be directly measured & Cannot be directly measured \\
\hline & For ideal gas & $U$ function of $T$ only & $U$ function of $T$ only \\
\hline & Link macro/micro & $U=\sum\left(E_{K}\right)+\sum\left(E_{P}\right)$ & $?$ \\
\hline \multirow{3}{*}{$\Delta U$} & Property & $\mathrm{d} U=\delta Q-P_{\text {ext }} \cdot \mathrm{d} V$ & $\mathrm{~d} U=\delta Q-P_{\text {int }} \cdot \mathrm{d} V$ \\
\hline & Reversible & $\Delta U=Q-P \cdot \Delta V$ & $\Delta U=Q-P \cdot \Delta V$ \\
\hline & Irreversible & $U\left(T_{1}\right)-U\left(T_{0}\right)=Q-\int P_{\text {ext }} \cdot \mathrm{d} v$ & $U\left(T_{2}\right)-U\left(T_{0}\right)=Q-\int P_{\text {int }} \cdot \mathrm{d} v$ \\
\hline
\end{tabular}

The (hot) body $A$ emits more heat than it receives.

By the same reasoning

\section{Chart 1. Similarities and differences about internal energy.}

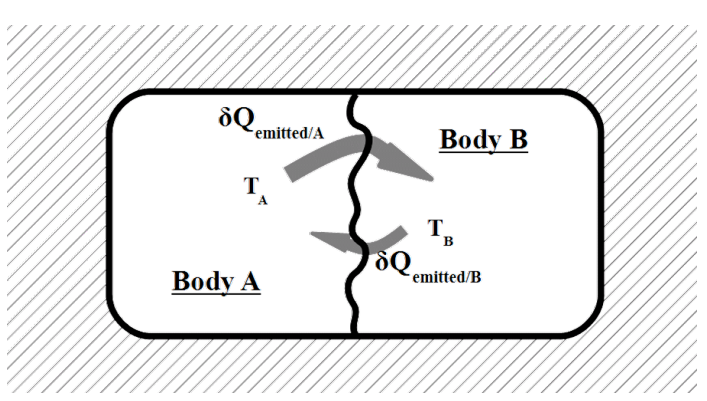

Figure 9. Heat transfer. 


$$
\delta Q_{\text {balance/B }}>0
$$

The (cold) body $B$ receives more heat than it emits.

Thus, the difference in heat is transferred from $A$ to $B$, or in other words:

The heat "difference" goes from hot to cold.

Result: That prohibits the perpetual motion machine of the second kind where heat is directly removed from a body: for there to be heat transfer we need a heat differential that goes from hot to cold.

\subsubsection{Case of Equilibrium}

When $T_{A}=T_{B}$, then

$$
\delta Q_{\text {balance } / A}=-\delta Q_{\text {balance } / B}=0
$$

When heat balance is nil, the bodies remain in thermal equilibrium. But that does not mean the heat exchanged is non-existent, which means the exchange is balanced. That is why it is distinguished. So we will distinguish the "balance heat" $\delta Q_{\text {balance/A }}(=0)$ from the "emitted heat" $\delta Q_{\text {emitted } / A}(\neq 0)$.

Using this argument on three bodies, mathematics proved that two temperatures equal to a third one are equal, and so the three bodies are in stable equilibrium; it would not be longer a thermodynamical principle, it would be a mathematical property.

\subsubsection{Extension}

For radiative exchange, according to Stéfan-Boltzmann’s law, the emittance $M$ of a black body is:

with $\sigma=5.67 \times 10^{-8}\left[\mathrm{~W} \cdot \mathrm{m}^{-2} \cdot \mathrm{K}^{-4}\right]$.

$$
M=\sigma T^{4}
$$

Let us have the hypothesis where the variable $s$ would be equal to $k T^{3}$ :

$$
s=k T^{3}
$$

Note in this hypothesis the variable $s$ increases as a function of temperature.

For a given area,

$$
\begin{gathered}
\delta Q_{\text {balance/A }}=\delta Q_{\text {emitted } / B}-\delta Q_{\text {emitted } / A}=k T_{B}^{4} \mathrm{~d} t-k T_{A}^{4} \mathrm{~d} t \\
\delta Q_{\text {balance/A }}=\left(T_{B}-T_{A}\right) k\left(T_{B}^{3}+T_{B}^{2} T_{A}+T_{B} T_{A}^{2}+T_{A}^{3}\right) \mathrm{d} t
\end{gathered}
$$

For $T_{A}$ and $T_{B}$ of the same order of magnitude, let us have $\varepsilon_{T}$ such as

$\mathcal{E}_{T}$ being small compared to $T_{A}$ and $T_{B}$.

$$
T_{A}=T_{B}+\varepsilon_{T},
$$

$$
\delta Q_{\text {balance/ } A}=\varepsilon_{T} \cdot k \cdot\left[4 T_{B}^{3}+\varepsilon_{T}\left(6 T_{B}^{2}+4 T_{B} \varepsilon_{T}+\varepsilon_{T}^{2}\right)\right] \mathrm{d} t
$$

$\varepsilon_{T}$ being small compared to $T_{B}$,

and with

$$
\delta Q_{\text {balance } / \mathrm{A}} \approx \varepsilon_{T} \cdot 4 \mathrm{k} \cdot T_{B}^{3} \cdot \mathrm{d} t
$$

$$
C=4 k \cdot T_{B}^{3} \cdot \mathrm{d} t
$$

and $\varepsilon_{T}=T_{A}-T_{B}$ according to Equation (23):

$$
\delta Q_{\text {balance/A }} \approx C\left(T_{B}-T_{A}\right)
$$

On a low temperature range and for a given duration $t, \delta Q_{\text {balance/A }}$ would be proportional to $\left(T_{B}-T_{A}\right)$ for radiative exchanges.

\subsection{Joule Experiment and Adiabatic Expansion}

\subsubsection{The Joule's Law and the Selected Experiments}

First we have to distinguish 
- the Joule's law: the internal energy depends only of the internal temperature for an ideal gas, from

- the so-called experiment of Joule: the adiabatic expansion in vacuum. It is a specific experiment because it is done against vacuum, and so it is irreversible. Let us remind experiments and industrial practice indicate that without heat transfer $(\delta Q=0)$ the gases cool by relaxing (at temperatures lower than the Mariotte temperature and at low pressure).

\subsubsection{Usual Explanations}

In the case of the expansion against vacuum, there is no heat exchange or external work exchange. Thus, according to Equation (14) $\mathrm{d} U=0$, so $U=$ Constant, and therefore (see Joule's law) $T=$ Constant. As this is in contradiction with the experimental results where the temperature is decreasing, it is said that the gases do not follow the ideal gas law [13]! Equation (1) of ideal gas would be invalid in these cases. Why would not rather Equation (2) of internal energy and so Equation (14) be invalid for irreversible transformations? See Figure 10 on incompatible assumptions.

Another explanation is to assert absolute vacuum does not exist (even in the intergalactic space, there is one particle per $\mathrm{m}^{3}$ ), there is always some molecules and thus a (tiny) level of external pressure $\zeta$, and therefore work of external forces:

$$
\begin{gathered}
\delta W_{\text {ext }}=-\zeta \cdot \mathrm{d} V_{\text {int }} \\
\mathrm{d} V_{\text {int }}>0 \text { so } \delta W_{\text {ext }} \leq 0 \text { and } \mathrm{d} U \leq 0
\end{gathered}
$$

In this case, the gas should hardly cool (whereas in practice, the gas really cools).

\subsubsection{Proposed Explanation}

According to Equation (16): $\mathrm{d} U=\delta Q-P_{\text {int }} \cdot \mathrm{d} V$. The internal pressure of the gas being never nil, the internal

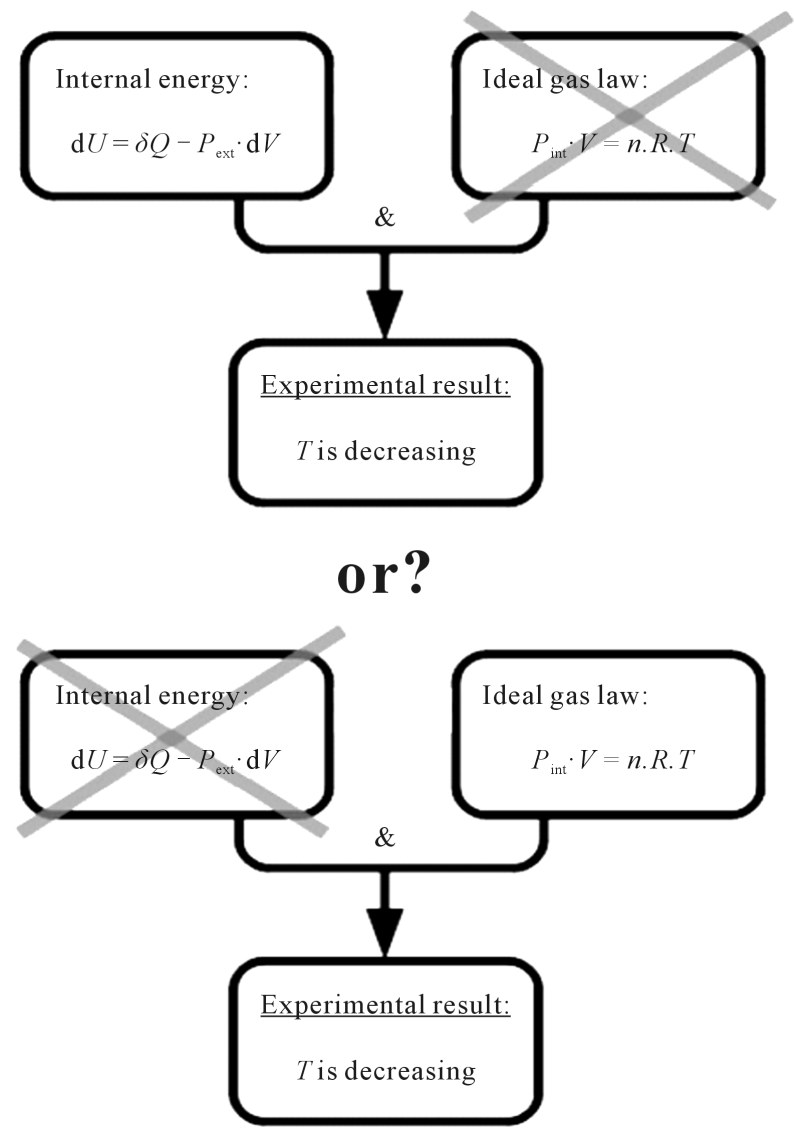

Figure 10. Incompatible assumptions. 
work is not nil, $\mathrm{d} V_{\text {int }}>0$, and $\delta Q=0$, therefore $\mathrm{d} U<0$ : placing itself under the usual conditions (lower temperature than Mariotte temperature and so to a pressure where the molecules do not interact), the ideal gas must cool itself by relaxing in accordance with the present hypothesis, and in accordance with experiments (without making necessarily a perfect vacuum).

A more precise calculation will be to use the formula from the Appendix:

$$
\mathrm{d} W_{\text {int. }}=\left(P_{\text {int. }}+P_{\text {ext. }}\right) / 2 \cdot \mathrm{d} V_{\text {int }} .
$$

Because $\delta Q=0$ and $P_{\text {ext }}=0$,

$$
\begin{gathered}
\mathrm{d} U=-P_{\text {int }} / 2 \cdot \mathrm{d} V_{\text {int }}, \\
\mathrm{d} U<0 \text { so } \mathrm{d} T<0 .
\end{gathered}
$$

The experimental results are in agreement with the Joule's law: with the expansion work the temperature is decreasing; and so there is a loss of internal energy (gained by the expansion work).

Conversely, in case of compression, the temperature increases and the internal energy consequently.

\subsection{The Area of Internal Pressure Work}

During an expansion the volume increases, and so according to Equation (13) where $\delta W_{\text {int }}=P_{\text {int }} \cdot d V_{\text {int }}$ : the internal work is positive $\left(W_{\text {int }}>0\right)$.

When the abscise increases for a positive function, the mathematical integral represented by its area is positive $\left(W_{\text {math }}>0\right)$. See Figure 11 .

The same reasoning can be applied to the volume restriction and to the negative integral.

There is total correlation between the thermo dynamical work of the internal pressure $W_{\text {int }}$ and the mathematical graphic representation $W_{\text {math }}$ in the Clapeyron diagram in [P-V].

\subsection{Engine Cycle}

"A transformation is reversible if it can return to its initial position by a series of infinitesimal changes. It is only a thought experiment: the actual transformations are all irreversible" [14].

The Figure 6 represents the idealized but incorrect case where heat is transferred whereas the internal fluid is at the same temperature as the hot source, and then at the same temperature as the cold source. For there to be heat transfer, "in practice, there must be some difference in temperature between the machine and the hot and cold sources" [15]. For the refrigerating machine, the fluid must be compressed to a temperature above the hot source, and then expanded to a temperature below the cold source for it to work. The Figure 6 should be reviewed as follows in Figure 12.

In refrigeration mode, heat of the cold source goes towards the colder fluid. Then the cycle fluid is compressed by the work done up to a temperature above the heat source for the heat dissipation of the fluid. And to close the cycle, the fluid is relaxed and cooled itself to return to the initial conditions.

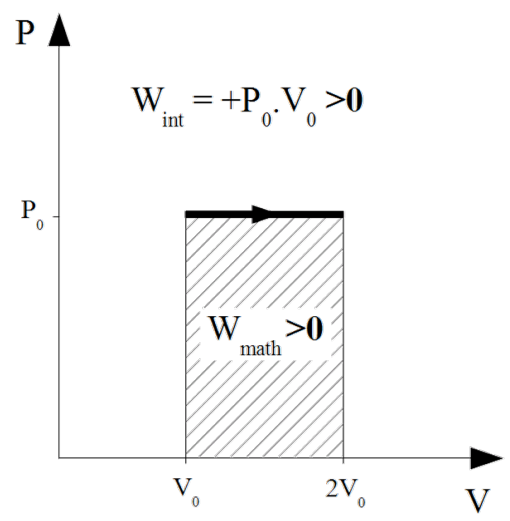

Figure 11. Representation of internal pressure work. 
The Figure 5 of the motor cycle are also idealized and incorrect, as there is no transfer of heat if the fluid is at the same temperature as the hot source, and then as the cold source. The Figure 5 should be reviewed as follows in Figure 13.

In motor mode, heat from the heat source goes to the cooler fluid, the fluid expansion provides work (and to complete the cycle, the fluid transfers the heat balance to the cold source).

\subsection{Motor Efficiency}

\subsubsection{Motor Efficiency Formula}

And Figure 4 would have to be reviewed as follows in Figure 14.

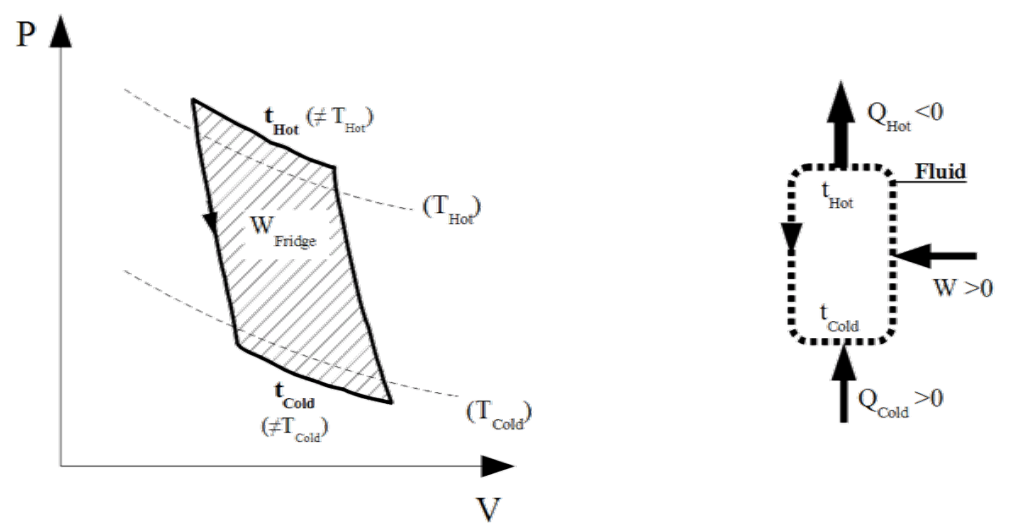

Figure 12. Proposed representation of cooling cycle.
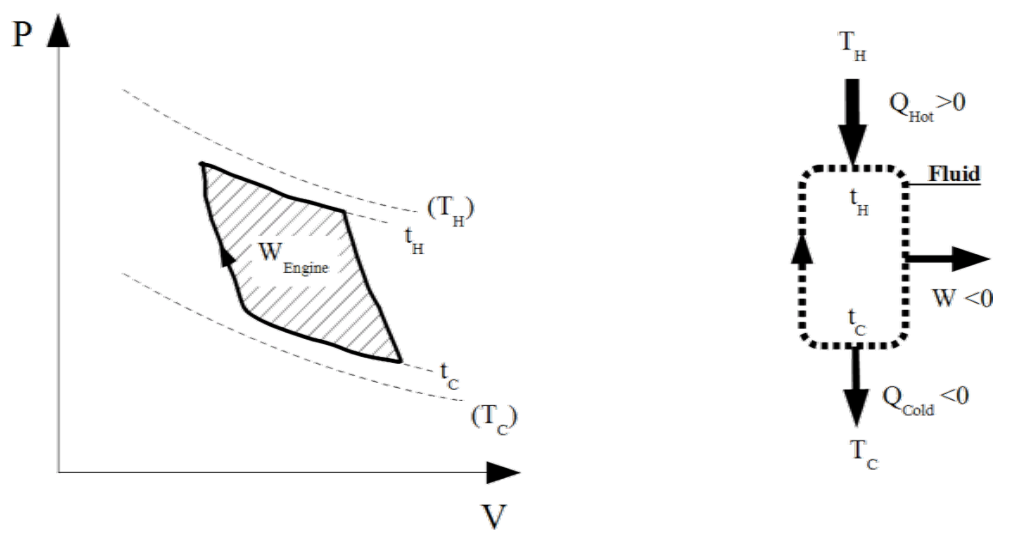

Figure 13. Proposed representation of motor cycle.

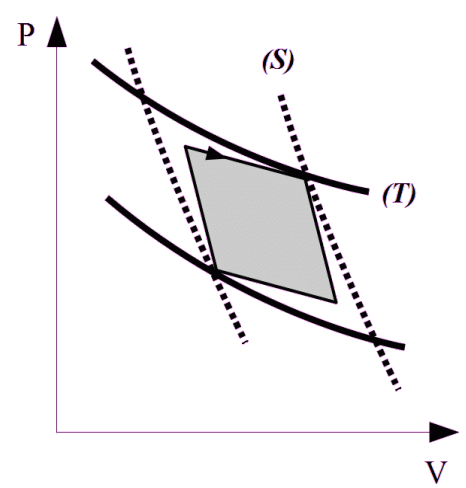

Figure 14. Proposed Carnot engine. 
If this cycle is more realistic, it will generate complicated equations to calculate variable temperatures. That is why it is suggested here the following simplification, knowing that $t_{H}$ and $t_{C}$ are approximately (not exactly) constant. Let us characterize it with equations:

The heat transferred to the (relative) hot fluid from the Hot source is (cf Equations (11) and (18)):

$$
Q_{\text {balance/hot fluid }}=s_{H} \cdot\left(T_{H}-t_{H}\right) \cdot t \text { for a given area }
$$

Note: the Hot source and the (relative) hot fluid being substantially at the same temperature, we consider firstly that they have substantially the same $s_{H}$ (idem for $s_{C}$ ). $\left.Q_{C}\right)$.

For writing formulas reason, let us simplify the expression: $Q_{\text {balance/hot fluid }}$ into $Q_{H}$ (and $Q_{\text {balance/cold fluid }}$ into

$$
\begin{gathered}
Q_{H}=s_{H} \cdot\left(T_{H}-t_{H}\right) \cdot t \text { for a given area } \\
\text { As } T_{H}>t_{H} \text { then } Q_{H}>0
\end{gathered}
$$

And the heat transferred from the (relative) cold fluid to the Cold source is [cf Equation (11) and Equation (19)]:

$$
\begin{gathered}
Q_{C}=s_{C} \cdot\left(T_{C}-t_{C}\right) \cdot t \text { for a given area } \\
\text { As } T_{C}<t_{C} \text { then } Q_{C}<0
\end{gathered}
$$

Preliminary remark: according that we adhere or not to the hypothesis expressed by Equation (15) taking into account internal work, demonstration hereafter does not change, it is why it is denoted by $|W|$, which can represent as well $\left|W_{\text {int }}\right|$ as $\left|W_{\text {ext }}\right|$.

For a motor cycle:

$$
Q_{H}=|W|+\left|Q_{C}\right|
$$

Motor efficiency $\eta$ is:

$$
\eta=\frac{|W|}{Q_{H}}
$$

or according to Equation (44):

$$
\eta=1-\frac{\left|Q_{C}\right|}{Q_{H}}
$$

and using equalities (40) and (42):

$$
\eta=1-\frac{s_{C}}{s_{H}} \cdot \frac{t_{C}-T_{C}}{T_{H}-t_{H}}
$$

On the same range of temperature, $s_{C} \cong s_{H}$, so

$$
\eta \cong 1-\frac{t_{C}-T_{C}}{T_{H}-t_{H}}
$$

which, for effective engine, is more accurate than the usual limit inequality $\eta \leq 1-\frac{T_{C}}{T_{H}} \quad$ [cf Equation (5)].

\subsubsection{Theoretical Example}

To get a better understanding, let us apply to a theoretical example, the irreversible Carnot engine, as described in Figure 15.

The effective efficiency is according to definition Equation (45):

$$
\eta_{\text {eff. }}=\frac{|W|}{Q_{H}}=\frac{100}{67}=60 \%
$$




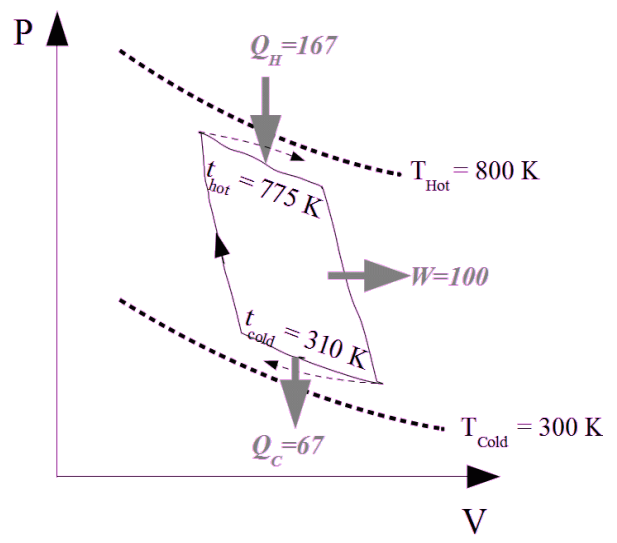

Figure 15. Irreversible Carnot engine.

If we use the efficiency of irreversible cycle based on temperatures as seen Equation (48):

$$
\eta_{\text {irrev. }} \cong 1-\frac{t_{C}-T_{C}}{T_{H}-t_{H}} \cong 1-\frac{310-300}{800-775} \cong 60 \%
$$

which has the same value than the effective efficiency,.

Thus the Carnot efficiency based on sources temperatures is, according to Equation (5):

$$
\eta \leq 1-\frac{T_{\text {Cold }}}{T_{\text {Hot }}}=1-\frac{300}{800}=62 \%
$$

which is slightly higher than the effective efficiency.

We can of course find the efficiency of $60 \%$ if we consider the cycle temperatures instead of the sources temperatures. The trouble is Carnot "engine" with isothermal evolution is unrealistic as explained §2.4. So, let us check another simple example.

\subsubsection{Application}

Then we will apply this property of the efficiency to a non-biphasic cycle: the very simple but more realistic elevator engine given by Pr. Richard Taillet [16]. Figure 16 and Chart 2 sum up the results:

In this non-biphasic cycle, the net work is $-45 \mathrm{~kJ}$ for a heat transfer of $(122+868=) 990 \mathrm{~kJ}$. The cycle effective efficiency is very low, $(45 / 990=) 5 \%$.

When we apply the Carnot efficiency, with a $746 \mathrm{~K}$ hot temperature and a $300 \mathrm{~K}$ cold temperature, the maximum efficiency is according to Equation (5):

$$
\eta_{\text {Carnot max. }} \leq 1-\frac{T_{C}}{T_{H}}=1-\frac{300}{746}=60 \%
$$

The maximum efficiency (60\%) based on sources temperatures is very far of this cycle efficiency (5\%).

To apply the new efficiency formula, we strictly should have used the instantaneous temperatures and integrate them, which would be very difficult here. So, only to give an order of magnitude, we will take in this example an average temperature of $((373+746) / 2=) 560 \mathrm{~K}$ for the hot side, and an average temperature of $((600+$ $300) / 2=) 450 \mathrm{~K}$ for the cold side. An irreversible efficiency should be closed to

$$
\eta_{\text {irrev. }} \cong 1-\frac{t_{C}-T_{C}}{T_{H}-t_{H}}=1-\frac{450-300}{746-560}=19 \%
$$

An irreversible efficiency (19\%) based on differences of temperatures is closer to the effective efficiency (5\%).

Previous calculation (19\%) was done with a very approximative but simple average temperature only to explain. It has also been taken final fluid temperatures equal to the source temperature, which would mean a perfect heat exchanger. In practice, we will get for example 4 to $5 \mathrm{~K}$ between the final fluid temperature and the 


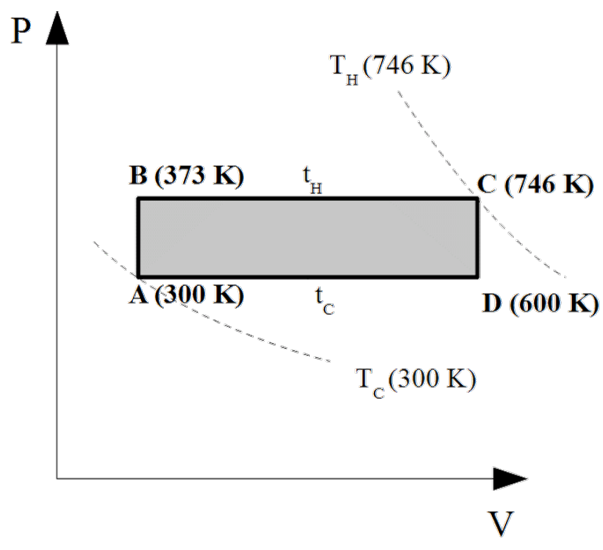

Figure 16. Non-biphasic motor cycle.

Chart 2. Cycle results.

\begin{tabular}{ccc}
\hline Exchanges & Heat $(\mathrm{kJ})$ & Work $(\mathrm{kJ})$ \\
\hline A-B & 122 & 0 \\
B-C & 868 & -245 \\
C-D & -245 & 0 \\
D-A & -700 & 200 \\
Cycle sum & 45 & -45 \\
\hline
\end{tabular}

source temperature: $\Delta \theta$. Let us use the more accurate logarithmic difference of temperature $\left(\Delta t_{l n}\right)$ used with heat exchangers:

$$
\Delta t_{l n}=\frac{(T+\Delta \theta-t)-\Delta \theta}{\ln ((T+\Delta \theta-t) / \Delta \theta)}
$$

which would give

$$
T_{H}-t_{H}=\Delta t_{l n / C}=\frac{(746+5-300)-5}{\ln ((746+5-300) / 5)}=99 \mathrm{~K}
$$

and, because $\Delta \theta$ is in practice a bit lower on the cold side than on the hot side

$$
t_{C}-T_{C}=\Delta t_{\ln / C}=\frac{(746-(300-4))-4}{\ln ((746-(300-4)) / 4)}=94 \mathrm{~K}
$$

so

$$
\eta_{\text {irrev. }} \cong 1-\frac{t_{C}-T_{C}}{T_{H}-t_{H}}=1-\frac{94}{99}=5 \%
$$

which is in good correlation with the $5 \%$ of the effective efficiency.

\section{Conclusion and Summary}

Slightly modifying the concepts of heat transfer and work, we have given different explanations, and hopefully clearer on:

- the thermal balance and the heat transfer direction;

- the effects of expansion in the so-called experiments of Joule;

- the direct reading of the work in the Clapeyron diagram; 
Chart 3. Comparison chart.

\begin{tabular}{|c|c|c|}
\hline Theory & Traditional & Proposed \\
\hline Heat received by the system & $\delta Q$ & $\delta Q_{\text {balance }}$ \\
\hline Equilibrium of 2 bodies & $Q=0$ & $Q_{\text {balance }}=0$ but $Q_{\text {emitted }} \neq 0$ \\
\hline Equilibrium of 3 bodies & by principle (\# 0) & as a consequence \\
\hline Heat goes from hot to cold & by principle (\# 2) & as a consequence \\
\hline Heat transfer from $A$ to $B$ & $Q_{A B}=C\left(T_{B}-T_{A}\right)$ by $C$ definition & $Q_{A B}=C\left(T_{B}-T_{A}\right)$ as a Boltzmann consequence \\
\hline Pressure work & $\Delta W=-P_{\text {ext }} \cdot d V_{\text {int }}$ & $\Delta W_{\text {int }}=+P_{\text {int }} \cdot d V_{\text {int }}$ \\
\hline Clapeyron diagram & $W$ and $W_{\text {math }}$ have opposite signs & $W_{\text {int }}$ and $W_{\text {math }}$ have same sign \\
\hline$U$ internal energy & State function & State function \\
\hline Joule’s law & $U$ function of $T$ & $U$ function of $T$ \\
\hline Energy variation & $\mathrm{d} U=\delta Q-P_{\text {ext }} \cdot \mathrm{d} V$ & $\mathrm{~d} U=\delta \mathrm{Q}-P_{\text {int }} \cdot \mathrm{d} V$ \\
\hline Adiabatic expansion & $\mathrm{d} U=0$ & $\mathrm{~d} U<0$ \\
\hline Adiabatic compression & $\mathrm{d} U=0$ & $\mathrm{~d} U>0$ \\
\hline Cycle efficiency definition & $\eta_{\text {cycle }}=\left|\frac{W}{Q_{H}}\right|$ & $\eta_{\text {cycle }}=\left|\frac{W}{Q_{H}}\right|$ \\
\hline Carnot cycle efficiency & $\eta_{\text {Camot cycle }}=1-\left|\frac{Q_{C}}{Q_{H}}\right|$ & $\eta_{\text {Camot cycle }}$ is not defined $\left(Q_{H}=Q_{C}=0\right)$ \\
\hline Irreversible engine efficiency & $\eta_{\text {irrev. }}=?$ & $\eta_{\text {irev. }} \cong 1-\frac{t_{C}-T_{C}}{T_{H}-t_{H}}$ \\
\hline Engine efficiency limit & $\eta_{\text {engine }} \leq 1-\frac{T_{C}}{T_{H}}$ & $\eta_{\text {engine }}<1-\frac{T_{C}}{T_{H}}$ \\
\hline
\end{tabular}

- a more accurate use of diagram of thermal cycles, especially for irreversible transformations in the motor cycle and the refrigeration cycle;

- a new property for irreversible engine efficiency: $\eta_{\text {irrev. }} \cong 1-\frac{t_{C}-T_{C}}{T_{H}-t_{H}}$.

You will find a chart (Chart 3) to sum up the proposed formulas vs. the traditional formulas.

Without questioning the experimental results and the reversible predictions, the purpose of this essay is contributing to evolve the presentation of the thermodynamics traditional explanations, taking better account of Irreversibility.

\section{Acknowledgements}

I would like to thank Daniel Mandineau for his teaching of traditional thermodynamics.

\section{References}

[1] Balian, R., CEA (2013) La longue élaboration du concept d'énergie. http://www.academie-sciences.fr/activite/hds/textes/evol Balian1.pdf 
[2] Diu, B. (2001) Quatre questions sur la thermodynamique. http://www.canal-u.tv/video/science_en_cours/quatre_questions_sur_la_thermodynamique_posees_a_bernard_diu.19

[3] ENS Cachan (1987) Gaz réels dilués, 140,141. http://www.physique.ens-cachan.fr/pagregp/enseignement/elec/corrigecompophy1987.pdf

[4] Rowlinson, J.S. (2009) James Joule, William Thomson and the Concept of a Perfect Gas. http://rsnr.royalsocietypublishing.org/content/64/1/43.full, extract: In 1845 Joule had tried to verify this assumption, which Thomson always called "Mayer's hypothesis", by expanding a cylinder of compressed gas into an empty cylinder but, as he later admitted, he failed because the heat capacity of his vessels was too large compared with that of the gas. We now know that for air at atmospheric pressure and temperature the difference between the two terms on the right of this equation is only about 3 parts per thousand of either of them.

[5] (1976) Détente, Larousse, http://www.larousse.fr/archives/grande-encyclopedie/page/4197 extract: The question arose whether the expansion of a gas in a vacuum, that is to say without external work, involving a change in temperature of the gas, assumed thermally insulated (expansion to constant internal energy); LJ Gay-Lussac then JP Joule have concluded in the negative, but their experiences were not very precise; GA Hirn (1865) brought to light a slight cooling.

[6] Dr. P. Puzo from Institut National de Physique Nucléaire (2012) Description des fluides réels, 127. https://users.lal.in2p3.fr/puzo/thermo/ch6_thermo.pdf

[7] Joule Expansion, Wikipedia. http://en.wikipedia.org/wiki/Joule expansion

[8] (2009) Gas Expansion Temperature Drop. http://www.oilngasseparator.info/gas-production-facility/hydrates/gas-expansion-temperature-drop.html

[9] Rapin, P., Jacquard, P. and Desmons, J. (2011) Technologie des Installations Frigorifiques, Edition Dunod, 492. Extract: The obtained cooling [of air] is in the order of $0.25^{\circ} \mathrm{C}$ for a pressure drop of 1 bar.

[10] Queyrel, J.-L. and Mesplede, J. (1996) Précis de Physique No. 6: Thermodynamique Prépas, Edition Bréal, 149.

[11] Trizac, E. (1999) Cours de Thermodynamique. http://www.lptms.u-psud.fr/membres/trizac/Ens/Livre_thermo.pdf

[12] Internal Energy, Wikipedia. http://en.wikipedia.org/wiki/Internal_energy

[13] Perez, J.-P. (2001) Détente de Joule et Gay-Lussac d’un gaz de Clausius. http://culturediff.pagesperso-orange.fr/jpp/ouvrage6/Thermo09-07.pdf

[14] Serres, M. and Farouki, N. (1997) Le Trésor-Dictionnaire des Sciences. Flammarion, 855.

[15] Gicquel, R., Mines de Paris Tech, Cycle de Carnot, p.4/9. http://direns.mines-paristech.fr/Sites/Thopt/fr/co/bases-thermodynamique.html

[16] Taillet, R., Université de Savoie (2012) Cours de thermodynamique. http://podcast.grenet.fr/episode/cours-7-2/ 


\section{Appendix}

\section{Arguments for Internal Pressure Work}

1) Work of external pressure

Because $\mathrm{d} V_{\text {ext }}=-\mathrm{d} V_{\text {int }}$,

$$
\delta W=-P_{\text {ext }} \cdot \mathrm{d} V_{\text {int }}=P_{\text {ext }} \cdot\left(-\mathrm{d} V_{\text {int }}\right)=P_{\text {ext }} \cdot d V_{\text {ext }}=\delta W_{\text {ext }}
$$

this means $\delta W$ is nothing but the work supplied (or received) by the outside to the (internal) system.

b) Work of internal pressure

More than the work supplied by the external, it would be more rigorous to take in count the work received (or supplied) by the (internal) system: $\delta W_{\text {int }}=P_{\text {int }} \cdot \mathrm{d} V_{\text {int }}$.

Due to the conservation of energy, the trouble is why the supplied work $\delta W_{\text {ext }}$ would not be equal to the received work $\delta W_{\text {int }}$ ? See Figure 17.

To get a movement, it is necessary to have $P_{\text {ext }} \neq P_{\text {int }}$, so $\delta W_{\text {ext }} \neq \delta W_{\text {int }}$.

3) Effective work

Effective work, supplied and received, is in fact

$$
\delta \mathrm{W}=\left(P_{\text {int }}+\alpha \cdot \mathrm{d} P\right) \cdot \mathrm{d} V_{\text {int }} \text { with } 0<\alpha<1
$$

this will be difficult to integrate

$$
W=\int\left(P_{\text {int }}+\alpha \cdot \mathrm{d} P\right) \cdot \mathrm{d} V_{\text {int }}
$$

So previous equation can be approximated by

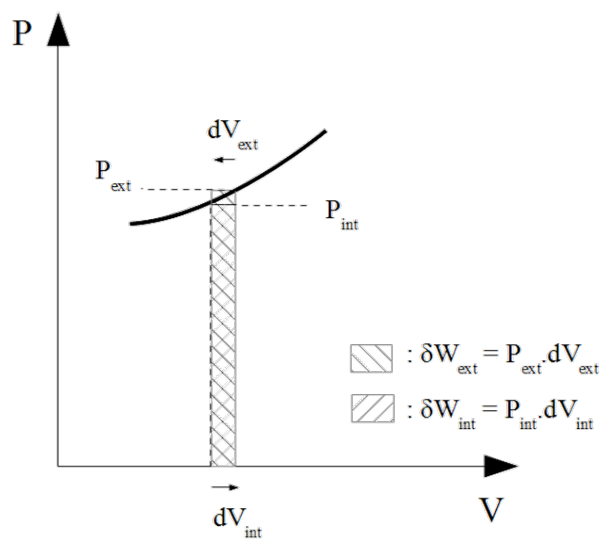

Figure 17. $W_{\text {ext }}$ and $W_{\text {int }}$.

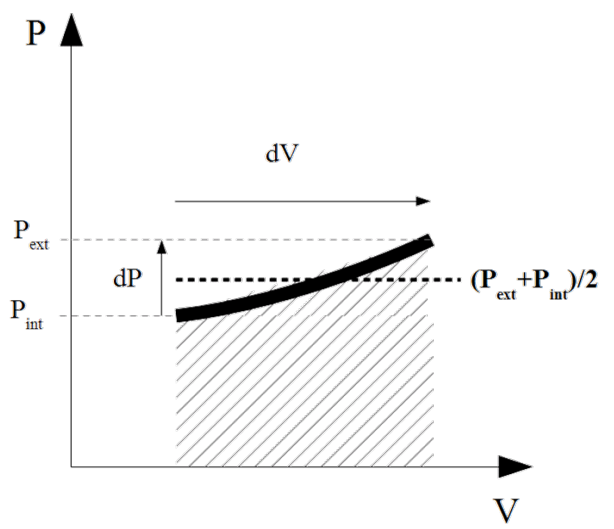

Figure 18. Effective work (supplied and received). 


$$
\delta W \approx\left(P_{\text {int }}+P_{\text {ext }}\right) / 2 \cdot \mathrm{d} V_{\text {int }}
$$

See Figure 18.

For pragmatic reasons (for example Clapeyron diagram, cf §4.3), it has been chosen here to represent $\delta W$ by $P_{\text {int }} \cdot \mathrm{d} V_{\text {int }}$, but if high precision is required, one of the two previous formulas [(A.4) or more precisely (A.2)] will be necessary. 
Scientific Research Publishing (SCIRP) is one of the largest Open Access journal publishers. It is currently publishing more than 200 open access, online, peer-reviewed journals covering a wide range of academic disciplines. SCIRP serves the worldwide academic communities and contributes to the progress and application of science with its publication.

Other selected journals from SCIRP are listed as below. Submit your manuscript to us via either submit@scirp.org or Online Submission Portal.
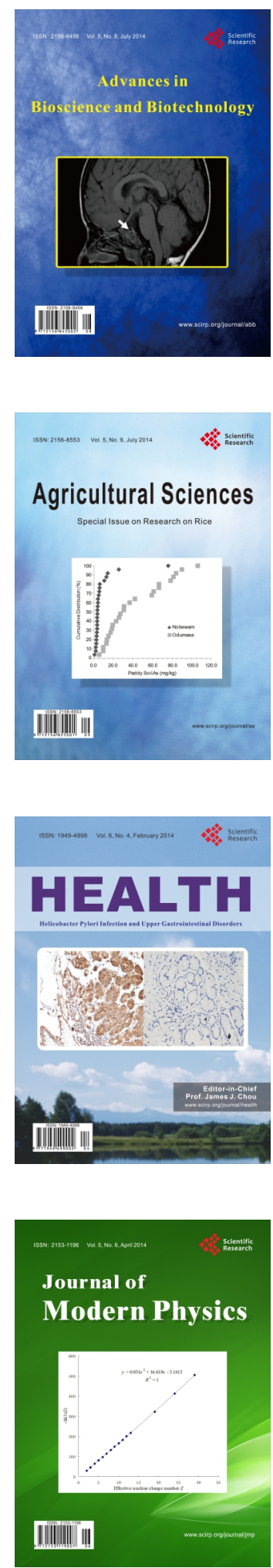
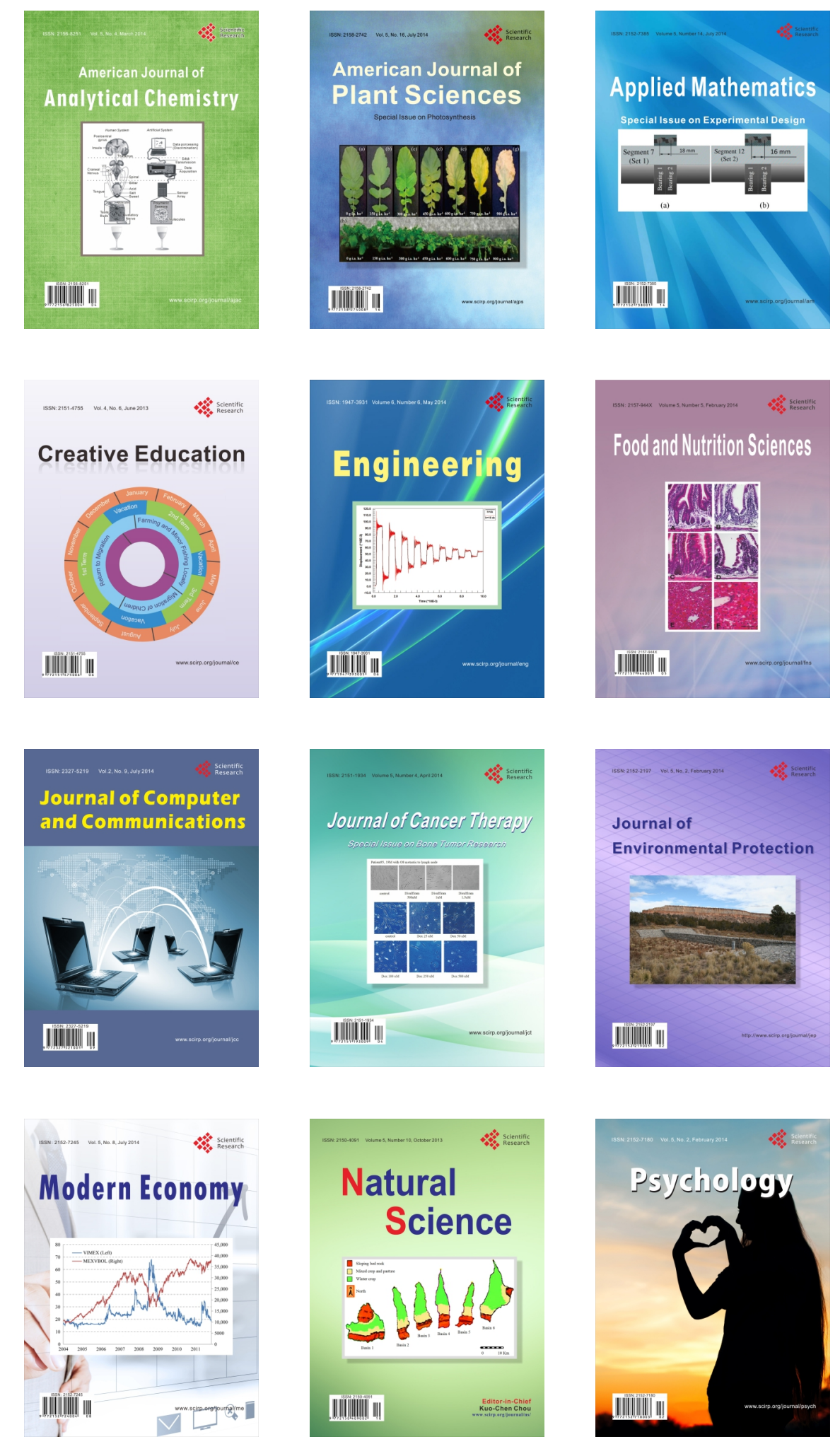